\title{
Does Integrative Medicine Enhance Balance in Aging Adults? Proof of Concept for the Benefit of Electroacupuncture Therapy in Parkinson's Disease
}

\author{
Nima Toosizadeh ${ }^{a}$ Hong Lei $^{b}$ Michael Schwenk $^{a, c}$ Scott J. Sherman ${ }^{b}$ \\ Esther Sternberg $^{d}$ Jane Mohler ${ }^{c}$ Bijan Najafia, ${ }^{c}$ \\ ${ }^{a}$ Interdisciplinary Consortium on Advanced Motion Performance (iCAMP) and Southern Arizona Limb Salvage \\ Alliance (SALSA), Department of Surgery, and ${ }^{b}$ Department of Neurology, College of Medicine, ${ }^{c}$ Arizona Center on \\ Aging, and ${ }^{\mathrm{d}}$ Arizona Center for Integrative Medicine, University of Arizona, Tucson, Ariz., USA
}

\section{Key Words \\ Parkinsonism · Acupuncture · Postural control · \\ Rigidity · Ankle strategy · Hip strategy · \\ Anterior-posterior sway $\cdot$ Medial-lateral sway}

\begin{abstract}
Background: Postural balance and potentially fall risk increases among older adults living with neurological diseases, especially Parkinson's disease (PD). Since conventional therapies such as levodopa or deep brain stimulation may fail to alleviate or may even worsen balance, interest is growing in evaluating alternative PD therapies. Objective: The purpose of the current study was to assess improvement in postural balance in PD patients following electroacupuncture (EA) as an alternative therapy. Methods: 15 aging adults (71.2 \pm 6.3 years) with idiopathic PD and 44 healthy agematched participants (74.6 \pm 6.5 years) were recruited. The PD participants were randomly assigned (at a ratio of $2: 1$ ) to an intervention $(n=10)$ or to a control group $(n=5)$. The intervention group received a 30-min EA treatment on a weekly basis for 3 weeks, while the control group received a sham treatment. Outcomes were assessed at baseline and
\end{abstract}

after the final therapy. Measurements included balance assessment, specifically the ratio of medial-lateral (ML) center-of-gravity (COG) sway to anterior-posterior (AP) sway $\left(\mathrm{COG}_{\mathrm{ML} / \mathrm{AP}}\right)$ and ankle/hip sway during eyes-open, eyesclosed, and eyes-open dual-task trials, the Unified Parkinson's Disease Rating Scale (UPDRS), as well as quality of life, concerns for fall, and pain questionnaires. Results: No difference was observed for the assessed parameters between the intervention and the control group at baseline. After treatment, an improvement in balance performance was observed in the intervention group. Compared with the healthy population, PD patients prior to treatment had larger $\mathrm{COG}_{\mathrm{ML} / \mathrm{AP}}$ sway with more dependency on upper-body movements for maintaining balance. Following EA therapy, $\mathrm{COG}_{\mathrm{ML} / \mathrm{AP}}$ sway was reduced by $31 \%$ and ankle/hip sway increased by $46 \%$ in the different conditions ( $p=0.02$ for the dual-task condition). The clinical rating revealed an overall improvement $(p<0.01)$ in mentation, behavior, and mood

The content of this article is solely the responsibility of the authors and does not necessarily represent the official views of the National Institute on Aging or the National Institutes of Health.

\section{KARGER 125}

(c) 2014 S. Karger AG, Basel

$0304-324 \mathrm{X} / 14 / 0611-0003 \$ 39.50 / 0$

E-Mail karger@karger.com

www.karger.com/ger
Bijan Najafi, PhD

Interdisciplinary Consortium on Advanced Motion Performance (iCAMP)

Department of Surgery, College of Medicine, University of Arizona

1501 N Campbell Ave \#4303D, Tucson, AZ 85724 (USA)

E-Mail bnajafi@surgery.arizona.edu 
(UPDRS part I, 49\%), activities of daily living (UPDRS part II, $46 \%$ ), and motor examination (UPDRS part III, 40\%). There was a significant reduction $(p<0.02)$ in the specific items regarding UPDRS fall status (67\%) and rigidity (48\%). Changes were small and nonsignificant in the controls $(p>0.29)$. Conclusions: This pilot study demonstrates improvement in rigidity and balance following EA. These preliminary results suggest EA could be a promising alternative treatment for balance disturbance in PD.

(c) 2014 S. Karger AG, Basel

\section{Introduction}

The number of older adults aged 65 years and more is projected to double by 2050 [1]. Despite extensive efforts for improving elders' quality of life, fall-induced injury is still a major source of morbidity and mortality [2]. In addition to aging, the risk of fall can increase in the presence of neurological diseases. Stolze et al. [3] reported that falls in neurological patients are twice as frequent as in agematched healthy populations, with the most frequently diagnosed falls in Parkinson's disease (PD) patients. Although a direct objective predictor of falling risk has not yet been discovered, several research studies have identified a strong association between poor postural balance and increased risk of falling. Abnormal postural sway measured by the range of sway, for example, has been introduced as a significant independent predictor of recurrent falls $[4,5]$, or as a distinguishable factor between fallers and nonfallers $[5,6]$.

$\mathrm{PD}$ is the second most common neurodegenerative disease worldwide, and is classically characterized by four cardinal features: tremor, bradykinesia, rigidity, and postural instability. Loss of balance control, which might significantly be associated with falls, is an inevitable consequence of the disease, and one of the most important mediators of quality of life $[7,8]$. Unfortunately, with common treatments such as levodopa or deep brain stimulation, balance control may not improve, or may even worsen [9-11]. In light of the significant limitations of conventional therapy, interest in complementary and alternative therapies for PD is growing. Exercise regimens and motor trainings, for example, have demonstrated significant enhancement in balance-related performance, functional mobility, and activities of daily living, and ultimately a reduction in risk of fall [12-15]. However, in the advanced stage of PD, patients may not be able to perform regular exercise. It is notable then that in addition to exercise as an alternative therapy for $\mathrm{PD}$, acupuncture is emerging as one of the most popu- lar of these alternatives [16-18]. Studies have demonstrated that acupuncture is the most frequent alternative treatment $[16,17]$, especially for improving sleep, quality of life, and motor performance $[18,19]$. However, in the majority of acupuncture studies, the quality of the outcome measures that can objectively identify relevant functional improvements is inadequate [19]. Most of the existing studies used subjective, global scales (e.g. the Unified Parkinson's Disease Rating Scale, UPDRS) covering a broad range of PD symptoms which are inappropriate to quantify specific changes such as compromised postural balance. Although wearable inertia sensors have been used to differentiate balance deficits in PD patients from those in the healthy population [20], to our knowledge, balance improvement in PD patients following acupuncture has not been objectively quantified using the wearable technology.

The purpose of the current study was to assess improvement in postural balance in PD patients following electroacupuncture (EA) therapy in a small sample of PD patients as a proof of concept. Poor balance control in PD patients compared to healthy populations has been previously linked to differences in direction of body sway and type of strategy (hip or ankle strategy). Compared to healthy older adults, PD patients may have more, or sometimes less, body sway during postural balance [21-23]; thus using total body sway is not reliable for measuring compromised balance in PD patients [24]. However, PD patients more consistently exhibit less anterior-posterior (AP) body sway and more medial-lateral (ML) sway as compared with age-matched healthy individuals [25]. Further, previous work suggests that PD patients have a higher tendency to use the hip joint for maintaining balance (hip strategy), instead of the ankle joints (ankle strategy), primarily as a result of increased ankle stiffness and a compromised reflexive response of the ankle muscles [26, 27]. Consequently, to test the efficacy of EA treatment in improving balance in PD participants, we hypothesized (1) a reduction in the ratio of ML/AP body sway and (2) an increased ratio of ankle rotations/hip rotations. As a secondary aim, we compared the ratio of ML/AP body sway and ankle/hip rotations between PD and healthy samples to confirm the differences between these two groups.

\section{Methods}

\section{Participants}

Aging adults aged 55 years or older with idiopathic PD diagnosed by movement disorder specialists based on the UK Brain Bank criteria were recruited from the University of Arizona Neurology Clinic. Participants were excluded if they were diagnosed
Toosizadeh/Lei/Schwenk/Sherman/ Sternberg/Mohler/Najafi 
with any type of neurological disorder other than PD or if they had prior experience of EA therapy. A healthy, nonfrail sample (measured by the Fried Index [28]) of community-dwelling older adults aged 55 years or older were recruited from University of Arizona geriatric clinics. As mentioned above, a large variability in balance behaviors exists among the PD population; therefore, healthy aging adults were recruited here as a sample in order to compare balance behaviors between healthy and $\mathrm{PD}$ groups prior to EA. Elders with major mobility or balance disorders, including PD, were excluded, as were those unable to walk a distance of 20 $\mathrm{m}$ without walking assistance. The study was approved by the University of Arizona Institutional Review Board, and written informed consent was obtained from all subjects before participation.

\section{Study Design}

The study was designed as a patient- and assessor-blinded, placebo-controlled, intervention pilot study. PD participants were randomly assigned (at a ratio of 2:1) to an intervention $(n=10)$ or a control group $(n=5)$. Since the primary focus of the current study was to explore balance improvement in PD patients following EA, a larger number of PD participants were allocated to the intervention group. Each participant in the intervention group went through a 30-min EA treatment once a week for 3 weeks, while participants in the control group received a weekly $30-\mathrm{min}$ sham treatment. Outcomes were assessed in the 'off-medication stage' ( $>12 \mathrm{~h}$ after the last PD medication dose) at baseline and after the final therapy. In addition, balance parameters prior to treatment were compared with an age-matched healthy population to assess impaired postural balance behaviors prior to treatment. To control for placebo effects, a similar experimental protocol was explained to all participants, and at the beginning of the experiment they were informed that they might be in either an intervention group or a control group.

Measurements included balance, the SF-12 health survey [29], the Short Falls Efficacy Scale-International (Short FES-I) [30], and a visual analog scale (VAS) for pain [31]. The UPDRS [32] was also performed by a trained examiner. Furthermore, participants were screened for cognition status using the Mini-Mental State Examination (MMSE) [33] and, for disease stage, using Hoehn and Yahr staging [34] at baseline. Fall status and rigidity were quantified at baseline and after treatment. Fall status was also quantified using subjective evaluation within the UPDRS, and rigidity with joint stiffness was assessed by the UPDRS examiner.

\section{EA Procedure}

A comprehensive and systematically applied EA regimen, consisting of scalp and body acupuncture, with a focus on improving balance and gait for PD patients, was designed and administered by H.L., who is double-certified by the American Board of Medical Acupuncture and the American Board of Psychiatry and Neurology. Sterile, disposable, surgical stainless steel acupuncture needles (L type, Seirin, Japan), measuring $0.25 \mathrm{~mm}$ in diameter and $40-50$ $\mathrm{mm}$ in length, were used for acupuncture, and electric stimulators (ITO ES-130, Japan) were used for EA stimulation. Real EA was performed at the following acupuncture points: GV20 (Baihui), GV14 (Dazhui) on the midline and bilateral foot motor sensory area, balance area, bilateral ST36 (Zusanli), LI4 (Hegu), GB34 (Yanglingquan), LR3 (Taichong), KI3 (Taixi), SP6 (Sanyinjiao), and BL40 (Weizhong). Electrical stimulation was applied for 30 min at a frequency of 4 or $100 \mathrm{~Hz}$ with an intensity just below the level that induces visible muscle contraction. The acupuncture points and frequencies were selected based on previous work [18, 19, 35, 36]. Sham acupuncture was performed for the control group with insertion of needles just under the skin at nonacupuncture points (the same for all subjects) at the scalp, neck, shoulder, and upper and lower extremities, and was stimulated in a similar fashion but with minimal intensity compared to real EA (just turning on the light of the stimulator). To assure that all participants in both the intervention and the control group received a similar sensation during the experimental procedure, the EA procedure was controlled to have the same duration with acupuncture needle placement as close as possible in both the intervention and the control group.

\section{Experimental Procedure}

Each participant performed six 30-second trials of balance assessment. In each trial, participants stood upright with their feet as close together as possible but without touching each other, and with their arms crossed. Participants were asked to cross their arms during balance tests to minimize the effect of arm movements on center-of-gravity (COG) displacements. In the first two trials, participants were instructed to keep their eyes open (eyes-open trials), with no target being specified. In the third and fourth trials, participants kept their eyes closed (eyes-closed trials). In the fifth and sixth trials, participants were instructed to count down from a random specified number while maintaining their balance with their eyes open (dual-task trials). In each trial the COG was estimated following identical procedures reported in our earlier study using wearable sensors $[37,38]$. Briefly, two sensors (fig. 1), each including a triaxial accelerometer, a triaxial gyroscope, and a triaxial magnometer, were used to estimate three-dimensional angles of the ankle and hip joints (BalanSens ${ }^{\mathrm{TM}}$; BioSensics LLC, Boston, Mass., USA). A two-link inverted-pendulum model of the human body was then used to calculate the COG from AP and ML angles during body movements.

\section{Balance Outcome Measures}

The outcome measures included body sway parameters: $\mathrm{COG}_{\mathrm{AP}}$ sway, $\mathrm{COG}_{\mathrm{ML}}$ sway, $\mathrm{COG}_{\mathrm{ML} / \mathrm{AP}}$ sway, ankle sway, hip sway, and ankle/hip sway. The sway ranges in AP and ML directions were defined as $\mathrm{COG}_{\mathrm{AP}}$ sway and $\mathrm{COG}_{\mathrm{ML}}$ sway, respectively, after excluding outliers, which were estimated by calculating 5 and 95 percentiles of the COG data (fig. 2) [37]. The $\mathrm{COG}_{\mathrm{ML} / \mathrm{AP}}$ sway was then calculated as the ratio of the sway range in ML over AP direction (i.e. $\mathrm{COG}_{\mathrm{ML} / \mathrm{AP}}$ sway $=\mathrm{COG}_{\mathrm{ML}}$ sway/COG $\mathrm{COP}_{\mathrm{AP}}$ sway). $\mathrm{COG}_{\mathrm{ML} / \mathrm{AP}}$ was measured here, since, according to previous studies, PD patients show larger $\mathrm{COG}_{\mathrm{AP}}$ sway and less $\mathrm{COG}_{\mathrm{ML}}$ sway than a healthy age-matched population $[21,25]$. As such, an increased value of $\mathrm{COG}_{\mathrm{ML} / \mathrm{AP}}$ sway demonstrates compromised balance in PD patients. Ankle sway was calculated as the product of the range of ankle rotations in the AP and ML directions; similarly, hip sway was calculated as the product of the ranges of hip rotation in the AP and ML directions. To evaluate improvement in using the ankle strategy instead of the hip strategy, the ratio of ankle sway over hip sway was calculated as ankle/hip sway. A larger magnitude of ankle/hip sway reveals an improvement in using the ankle joints for maintaining balance in PD patients. 


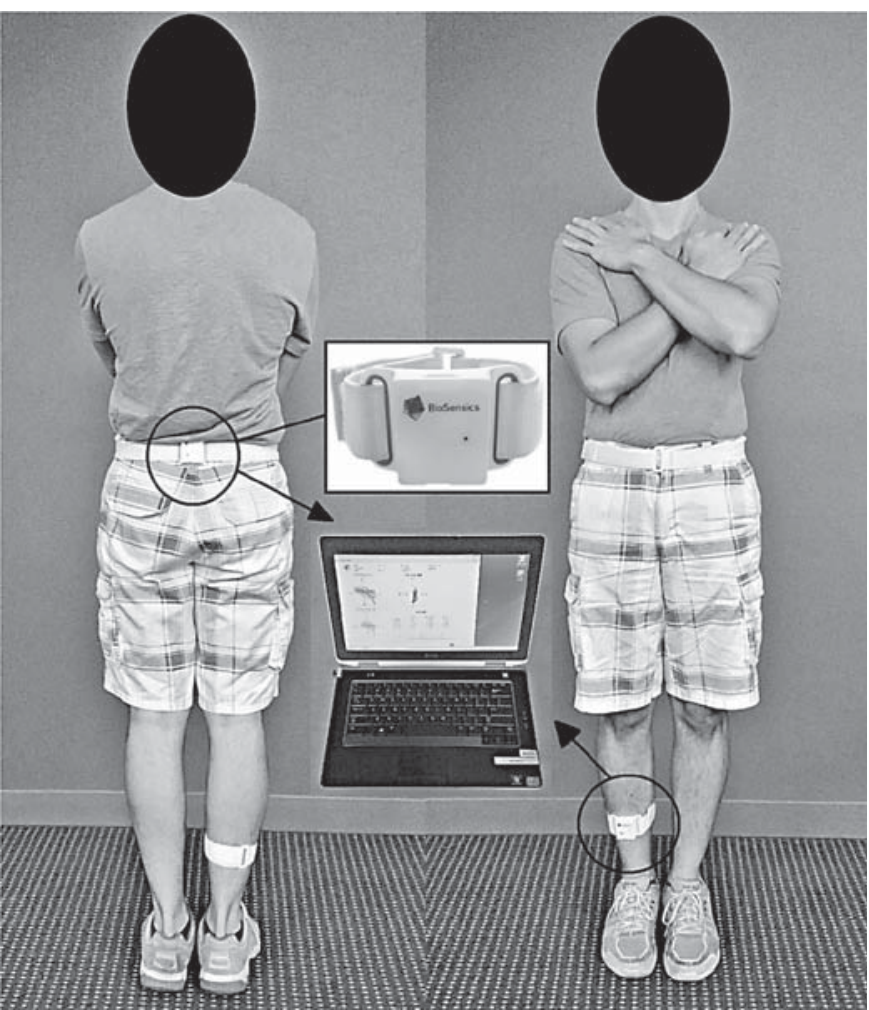

Fig. 1. Two wireless sensors are attached to the shank and trunk to provide three-dimensional angles of ankle and hip joints in real time, and to ultimately estimate COG.

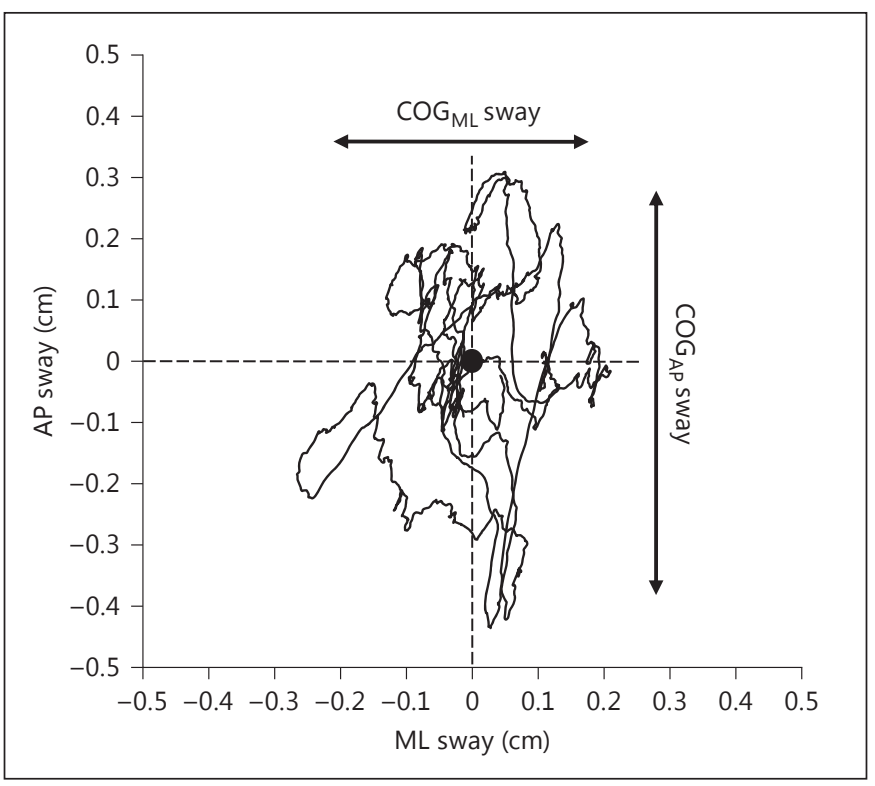

Fig. 2. Sample of a stabilogram plot from a nonfrail participant. The figure illustrates a larger body sway in the AP than in the ML direction.

\section{Statistical Analysis}

The comparison between the healthy and the PD group for baseline balance parameters was performed using the unpaired t test (or Mann-Whitney U test for nonparametric samples). Comparisons between the intervention and the control group for participants' characteristics and study outcomes were also performed using the unpaired t test (or Mann-Whitney U test for nonparametric samples) except for participants' gender, for which the $\chi^{2}$ test was used. Effects of EA on balance data were compared using the paired $t$ test (or Mann-Whitney $U$ test for nonparametric samples) for the intervention and the control PD group by comparing pre- and post-EA data.

Between-group (intervention vs. control) comparisons of changes in parameters were performed using mixed ANOVA models, considering age and body mass index (BMI) as covariates. The association between participants' characteristics and improvement in outcome measures following EA was assessed using linear regression models. Only parameters with the highest effect size were considered as dependent variables here, and baseline values of each parameter, age, disease stage, and BMI were used, in separate models, as independent variables. Summary results are presented as means $[ \pm$ standard errors (SE) or standard deviation (SD)]. All analyses were done using JMP ${ }^{\mathrm{TM}}$ version 10 (SAS Institute, Inc., Cary, N.C., USA), and statistical significance was concluded when $\mathrm{p}<0.05$.

\section{Results}

\section{Participants}

Fifteen participants with PD (age $71.2 \pm 6.3$ years; BMI $27.5 \pm 6.5 \mathrm{~kg} / \mathrm{m}^{2}$ ) and 44 healthy aging adults (age $74.6 \pm$ 6.5 years; BMI $25.7 \pm 4.5 \mathrm{~kg} / \mathrm{m}^{2}$ ) were recruited. Demographic information on the participants is reported in tables 1 and 2 .

\section{Balance: Comparison between the Healthy and the PD Sample}

Comparing the PD and the healthy sample showed no significant difference in demographic information, except for gender and MMSE score (table 2). Although these differences were observed, two groups were still considered demographically comparable, since no significant effect of gender or MMSE score was observed in balance outcome measures in either the healthy or the PD group ( $p>0.17)$. Overall, a larger amount of sway was noticeable among the healthy group compared to the PD participants; $\mathrm{COG}_{\mathrm{AP}}$, ankle, and hip sway were significantly larger among the healthy population by 116,125 , and $134 \%$, respectively $(\mathrm{p}<0.01)$, while $\mathrm{COG}_{\mathrm{ML}}$ sway was comparable between the two groups $(\mathrm{p}=0.77)$. $\mathrm{COG}_{\mathrm{ML} / \mathrm{AP}}$ sways were also significantly smaller (144\%) among the healthy group compared to the $\mathrm{PD}$ group $(\mathrm{p}<0.0001)$. 
Table 1. Mean (SD) values of participant demographic information and pre-EA balance behaviors
Table 2. Mean (SD) values of participant demographic information

\begin{tabular}{|c|c|c|c|c|}
\hline & $\begin{array}{l}\text { Inter- } \\
\text { vention }\end{array}$ & Control & Total & $\begin{array}{l}\mathrm{p} \\
\text { value }\end{array}$ \\
\hline Number (\% of total) & $10(67 \%)$ & $5(33 \%)$ & 15 & - \\
\hline Male, $\mathrm{n}$ (\% of the group) & $6(60 \%)$ & $2(40 \%)$ & $8(53 \%)$ & 0.46 \\
\hline Age, years & $71.1(3.0)$ & $71.4(10.9)$ & $71.2(6.3)$ & 0.78 \\
\hline Stature, $\mathrm{cm}$ & $164.6(10.1)$ & $163.6(13.5)$ & $164.3(10.9)$ & 0.87 \\
\hline Body mass, kg & $74.3(10.4)$ & $76.4(23.8)$ & $74.9(15.3)$ & 0.81 \\
\hline BMI, kg/m² & $27.5(4.1)$ & $28.1(6.5)$ & $27.5(6.5)$ & 0.85 \\
\hline MMSE score & $24.9(6.7)$ & $27.2(1.1)$ & $25.7(5.6)$ & 0.47 \\
\hline Disease stage & $3.0(1.0)$ & $2.9(0.7)$ & $2.9(0.9)$ & 0.92 \\
\hline $\mathrm{COG}_{\mathrm{AP}}$ sway, $\mathrm{cm}^{\mathrm{a}}$ & $0.50(0.34)$ & $0.69(0.51)$ & $0.61(0.40)$ & $0.38^{\mathrm{b}}$ \\
\hline $\mathrm{COG}_{\mathrm{ML}}$ sway, $\mathrm{cm}^{\mathrm{a}}$ & $0.61(0.39)$ & $0.82(0.20)$ & $0.70(0.41)$ & $0.18^{\mathrm{b}}$ \\
\hline $\mathrm{COG}_{\mathrm{ML} / \mathrm{AP}}$ sway $^{\mathrm{a}}$ & $1.42(0.60)$ & $1.66(0.86)$ & $1.54(0.68)$ & $0.42^{\mathrm{b}}$ \\
\hline Ankle sway, ${ }^{\circ}$ & $2.83(3.48)$ & $3.91(2.54)$ & $3.50(3.82)$ & $0.18^{\mathrm{b}}$ \\
\hline Hip sway, oa & $1.81(1.93)$ & $2.44(0.98)$ & $2.19(2.05)$ & $0.11^{b}$ \\
\hline Ankle/hip sway ${ }^{\mathrm{a}}$ & $1.38(0.43)$ & $1.59(0.75)$ & $1.50(0.53)$ & $0.26^{\mathrm{b}}$ \\
\hline UPDRS - fall & $0.9(1.3)$ & $0.8(0.8)$ & $0.9(1.1)$ & 0.88 \\
\hline UPDRS - rigidity & $6.4(5.1)$ & $7.4(4.5)$ & $6.7(4.8)$ & 0.72 \\
\hline UPDRS I & $5.3(3.5)$ & $3.3(2.4)$ & $4.6(3.2)$ & 0.28 \\
\hline UPDRS II & $18.0(9.7)$ & $17.0(5.7)$ & $17.7(8.4)$ & 0.84 \\
\hline UPDRS III & $35.1(15.3)$ & $34.2(12.3)$ & $34.8(13.9)$ & 0.91 \\
\hline
\end{tabular}

${ }^{a}$ Average values in different conditions (i.e. eyes-open, eyes-closed, and dual-task trials) were compared between intervention and control samples. ${ }^{\mathrm{b}}$ Smallest $\mathrm{p}$ value in the conditions (i.e. eyes-open, eyes-closed, and dual-task trials).

\begin{tabular}{lcccc}
\hline & PD & Healthy & 95\% CI & p value \\
\hline Number (\% of total) & 15 & 44 & - & - \\
Male, n (\% of the group) & $8(53 \%)$ & $7(16 \%)$ & 0.26 to 1.57 & $<0.01^{*}$ \\
Age, years & $71.2(6.3)$ & $74.6(6.5)$ & -0.21 to 3.71 & 0.12 \\
Stature, cm & $164.3(10.9)$ & $159.1(6.8)$ & -0.04 to 0.01 & 0.29 \\
Body mass, kg & $74.9(15.3)$ & $66.1(15.8)$ & -7.92 to 0.15 & 0.06 \\
BMI, kg/m & $27.5(6.5)$ & $25.7(4.5)$ & -2.38 to 0.36 & 0.15 \\
MMSE score $_{\text {COG }}$ sway, cm $_{\text {COG }}$ sway, cm & $25.7(5.6)$ & $29.2(1.1)$ & 0.64 to 1.52 & $<0.0001^{*}$ \\
COG $_{\text {ML/AP sway }}$ & $0.61(0.40)$ & $1.32(0.68)$ & 0.22 to 0.52 & $<0.0001^{*}$ \\
Ankle sway, $^{\circ}$ & $0.70(0.41)$ & $0.67(0.35)$ & -0.12 to 0.09 & 0.77 \\
Hip sway, $^{\circ}$ & $1.54(0.68)$ & $0.57(0.32)$ & -0.52 to -0.31 & $<0.0001^{*}$ \\
Ankle/hip sway & $3.50(3.82)$ & $7.87(6.85)$ & 0.63 to 3.88 & $<0.01^{*}$ \\
& $2.19(2.05)$ & $5.13(3.73)$ & 0.65 to 2.26 & $<0.001^{*}$ \\
\hline
\end{tabular}

For balance parameters, mean (SD) values across eyes-open and eyes-closed trials are presented. * Significant difference.
Balance: Comparison between the Intervention and the Control Group in the PD Sample

No significant difference was observed in participants' demographic information, disease stage, or MMSE score between the intervention and the control group $(p>0.47)$ (see table 1 for details). Further, the comparison of outcome measures (balance and subjective evaluations) at baseline showed no significant difference between the intervention and the control group $(p>0.11)$. After treatment, improvement in balance performance was ob- 
Fig. 3. Ratio of $\mathrm{COG}_{\mathrm{ML} / \mathrm{AP}}$ and ankle/hip sway in the intervention and the control group before EA (gray columns) and after EA (open columns) during eyes-closed and dual-task conditions. Mean values and SE are illustrated. * Significant difference.

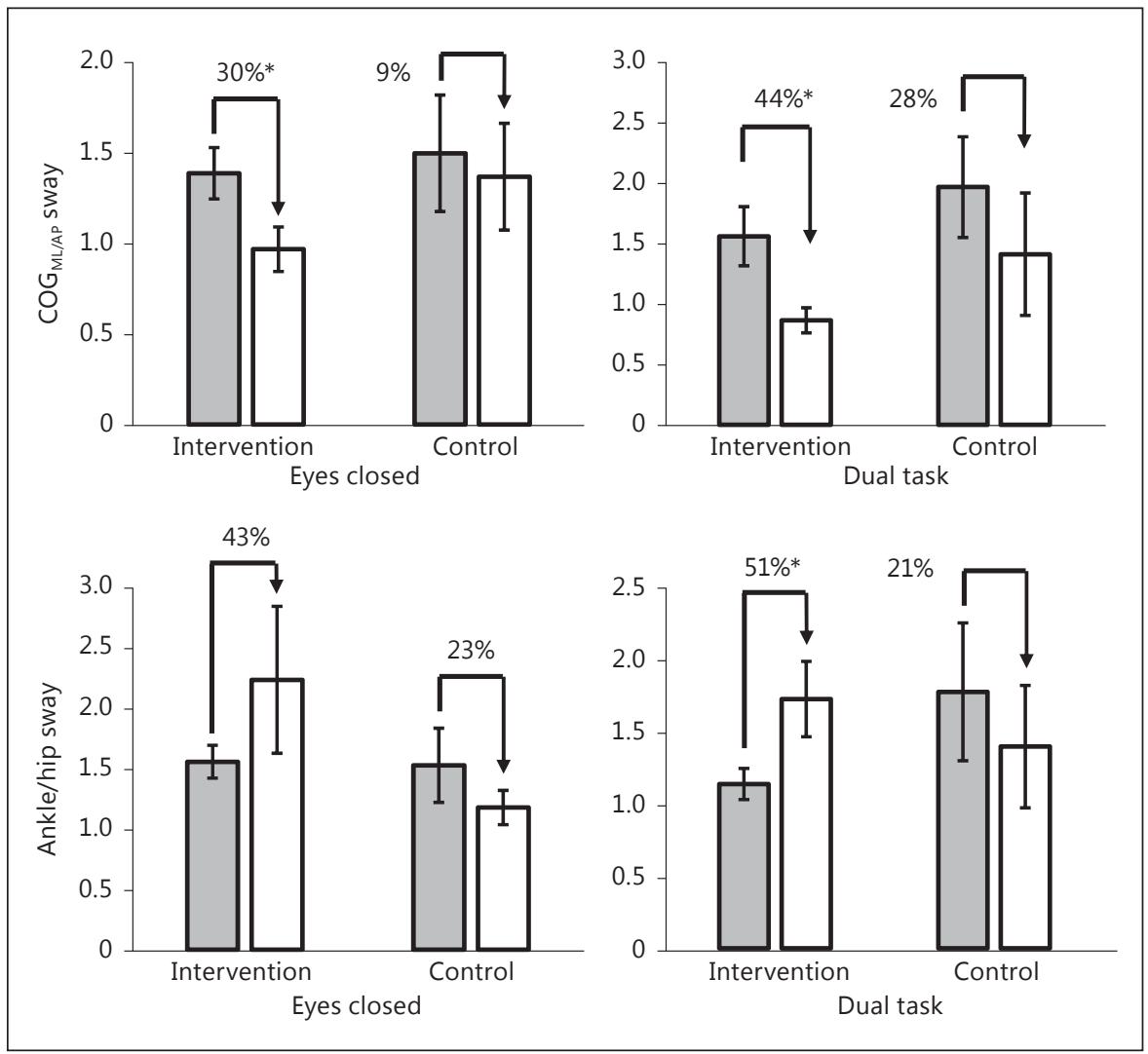

served in the intervention group; overall, $\mathrm{COG}_{\mathrm{ML} / \mathrm{AP}}$ sway was reduced by $31 \%$, and ankle/hip sway increased by $46 \%$ in the different conditions (see fig. 3 and table 3 for details). The pre-to-post-EA difference in the intervention group was more pronounced in the dual-task condition. Although improvement in balance performance was also observed in some of the outcome measures in the control group, the changes were small and nonsignificant ( $p$ > 0.29). Comparing balance parameter improvement between the intervention and the control group showed that improvement in balance parameters was significant only for ankle/hip sway $(\mathrm{p}=0.02)$ (table 4$)$. Regression models, however, showed no significant correlation ( $\mathrm{p}>$ 0.13 and $\mathrm{R}^{2}<27$ ) between ankle/hip sway improvement and disease stage or baseline values of ankle/hip sway.

\section{Subjective Evaluations and UPDRS Scores:}

Comparison between the Intervention and the Control Group in the PD Sample

The clinical rating revealed significant improvements $(\mathrm{p}<0.01)$ in mentation, behavior, and mood (UPDRS part I, 49\%), activities of daily living (UPDRS part II, 46\%), and motor examination (UPDRS part III, 40\%). There was a significant reduction $(\mathrm{p}<0.02)$ in the specific items regarding fall status (67\%) and rigidity (48\%) (table 5). Although the changes were not significant, the Short FES-I and VAS scores were also reduced by 15 and $44 \%$ following treatment in the intervention group $(p>0.26)$. No significant improvement was observed in the control group between before and after EA ( $p>0.27)$. The comparison between the intervention and the control group for improvement in UPDRS scores showed significant differences in rigidity, UPDRS I, UPDRS II, and UPDRS III ( $\mathrm{p}<$ 0.05) (table 4). Furthermore, the results from the regression models demonstrated significant positive correlations (fig. 4) between UPDRS III improvement and disease stage $(\mathrm{r}=0.65, \mathrm{p}=0.04)$, and between UPDRS III improvement and UPDRS III baseline values $(\mathrm{r}=0.81, \mathrm{p}<0.01)$.

\section{Discussion}

\section{Alterations in Body Sway following EA}

In confirmation of our hypotheses, we observed improvements in postural balance in PD patients following EA. Overall, the results demonstrate an increased sway fol- 
Table 3. Mean (SD) and pre-to-post-EA comparison of balance parameters in eyes-open, eyes-closed, and dual-task conditions for the intervention and the control group

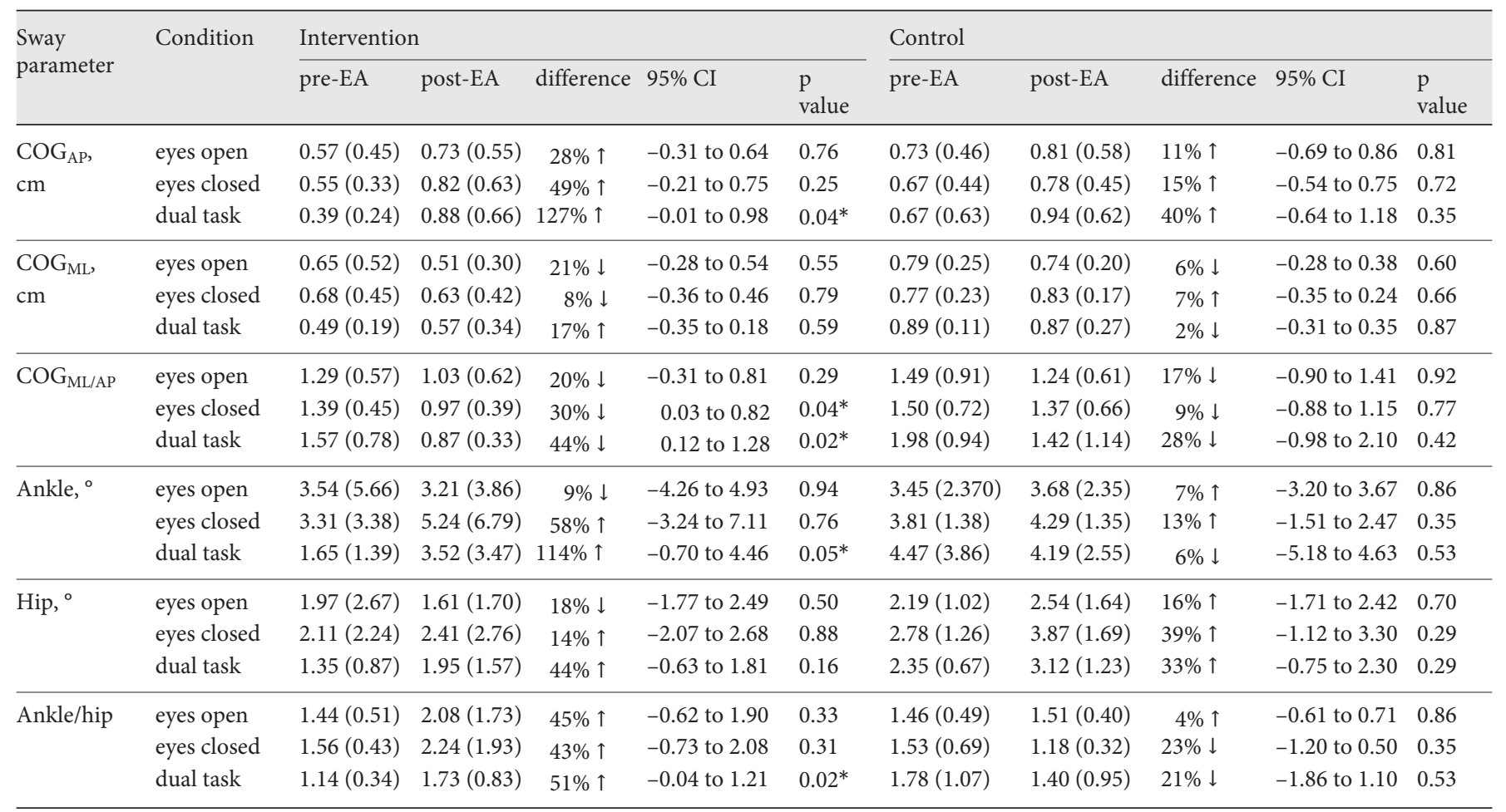

* Significant difference.

lowing EA, especially in the AP direction. Comparing PD patients with a control age-matched group, Horak et al. [21] observed that PD patients had a very small sway area, and related this small sway to a larger joint and muscle stiffness and overall 'rigidity' in PD patients. Conversely, other studies showed larger amounts of sway (area and velocity of sway) in PD patients compared to healthy age-matched groups $[22,23]$. According to previous evidence, combinations of the cardinal motor signs may differ between PD patients; one PD patient might develop joint rigidity, while limb tremor is more noticeable for another patient [39]. This may lead to a bimodal balance pattern (either very large or very small sway) in PD patients. To account for this variability in balance among those with $\mathrm{PD}$, we compared balance parameters with an age-matched healthy population prior to treatment initiation. Our comparison demonstrated a smaller sway in $\mathrm{PD}$ patients in the AP direction. After EA, $\mathrm{COG}_{\mathrm{AP}}$ sway increased by up to $127 \%$, to a value closer to those of healthy individuals, which can be considered a significant improvement in balance among those in the intervention group.
Other studies have suggested that the interaction or relationship between ML and AP sway is a more valid measure of balance impairment, especially in PD patients $[25,40]$. Healthy adults show a larger AP than ML sway $\left(\mathrm{COG}_{\mathrm{ML} / \mathrm{AP}}<0.6\right.$ in different conditions; see table 2$)$. In the current study, we observed that PD patients had a large mean (SD) $\mathrm{COG}_{\mathrm{ML} / \mathrm{AP}}$ sway ratio of $1.5(0.7)$ prior to the treatment, with an increasing trend by switching from eyes-open to eyes-closed, and from eyes-closed to dual-task conditions. The mean (SD) value of $\mathrm{COG}_{\mathrm{ML} / \mathrm{AP}}$ sway was reduced to $0.9(0.5)$ in the intervention group following EA treatment. The observed $\mathrm{COG}_{\mathrm{ML} / \mathrm{AP}}$ sway improvement can be explained based on the structure of the lower extremity joints and their corresponding balance control mechanism.

An improvement in $\mathrm{COG}_{\mathrm{ML} / \mathrm{AP}}$ sway could result from either increased $\mathrm{COG}_{\mathrm{AP}}$ sway or a reduction in $\mathrm{COG}_{\mathrm{ML}}$ sway; however, overall, an increase in $\mathrm{COG}_{\mathrm{AP}}$ sway was dominant here in $\mathrm{COG}_{\mathrm{ML} / \mathrm{AP}}$ sway improvement. Comparing $\mathrm{COG}_{\mathrm{AP}}$ and $\mathrm{COG}_{\mathrm{ML}}$ sway, previous studies have suggested that in healthy individuals body sway is larger in the AP than in the ML direction, mainly due to the in- 
Table 4. Mean (SD) changes after the treatment in balance and UPDRS parameters in the intervention and the control group

\begin{tabular}{|c|c|c|c|c|c|}
\hline Parameter & Intervention & Control & $95 \% \mathrm{CI}$ & $\mathrm{p}$ value & Effect size \\
\hline $\mathrm{COG}_{\mathrm{AP}}$ sway - eyes open & $0.2(0.6) \uparrow$ & $0.1(0.8) \uparrow$ & -1.0 to 0.9 & 0.85 & 0.14 \\
\hline $\mathrm{COG}_{\mathrm{AP}}$ sway - eyes closed & $0.3(0.6) \uparrow$ & $0.1(0.5) \uparrow$ & -0.9 to 0.5 & 0.60 & 0.36 \\
\hline $\mathrm{COG}_{\mathrm{AP}}$ sway - dual task & $0.5(0.7) \uparrow$ & $0.3(0.4) \uparrow$ & -0.8 to 0.4 & 0.42 & 0.37 \\
\hline $\mathrm{COG}_{\mathrm{ML}}$ sway - eyes open & $0.1(0.3) \downarrow$ & $0.1(0.3) \downarrow$ & -0.3 to 0.4 & 0.61 & 0.30 \\
\hline $\mathrm{COG}_{\mathrm{ML}}$ sway - eyes closed & $0.1(0.5) \downarrow$ & $0.1(0.3) \uparrow$ & -0.4 to 0.6 & 0.63 & 0.49 \\
\hline $\mathrm{COG}_{\mathrm{ML}}$ sway - dual task & $0.1(0.3) \uparrow$ & $0.1(0.2) \downarrow$ & -0.4 to 0.2 & 0.47 & 0.78 \\
\hline $\mathrm{COG}_{\mathrm{ML} / \mathrm{AP}}$ sway - eyes open & $0.3(0.7) \downarrow$ & $0.3(1.2) \downarrow$ & -1.4 to 1.4 & 0.99 & 0.01 \\
\hline $\mathrm{COG}_{\mathrm{ML} / \mathrm{AP}}$ sway - eyes closed & $0.4(0.6) \downarrow$ & $0.1(0.6) \downarrow$ & -0.5 to 1.5 & 0.41 & 0.50 \\
\hline $\mathrm{COG}_{\mathrm{ML} / \mathrm{AP}}$ sway - dual task & $0.7(0.7) \downarrow$ & $0.6(0.8) \downarrow$ & -0.8 to 1.1 & 0.74 & 0.13 \\
\hline Ankle sway - eyes open & $0.3(5.1) \downarrow$ & $0.2(2.8) \uparrow$ & -3.8 to 5.0 & 0.78 & 0.12 \\
\hline Ankle sway - eyes closed & $1.9(6.5) \uparrow$ & $0.5(2.2) \uparrow$ & -6.4 to 3.5 & 0.54 & 0.51 \\
\hline Ankle sway - dual task & $1.9(3.3) \uparrow$ & $0.3(2.1) \downarrow$ & -5.2 to 0.9 & 0.15 & 0.79 \\
\hline Hip sway - eyes open & $0.4(2.2) \downarrow$ & $0.4(1.6) \uparrow$ & -1.5 to 2.9 & 0.50 & 0.42 \\
\hline Hip sway - eyes closed & $0.3(3.4) \uparrow$ & $1.1(2.5) \uparrow$ & -2.7 to 4.2 & 0.63 & 0.27 \\
\hline Hip sway - dual task & $0.6(1.6) \uparrow$ & $0.8(1.4) \uparrow$ & -1.6 to 2.0 & 0.83 & 0.13 \\
\hline Ankle/hip sway - eyes open & $0.6(1.5) \uparrow$ & $0.1(0.4) \uparrow$ & -1.7 to 0.5 & 0.28 & 0.46 \\
\hline Ankle/hip sway - eyes closed & $0.7(1.8) \uparrow$ & $0.3(0.5) \downarrow$ & -2.4 to 0.4 & 0.06 & 0.76 \\
\hline Ankle/hip sway - dual task & $0.6(0.7) \uparrow$ & $0.4(0.6) \downarrow$ & -1.8 to 0.2 & $0.02 *$ & 1.53 \\
\hline SF-12 (PCS) & $0.6(6.4) \uparrow$ & $1.0(7.5) \downarrow$ & -10.8 to 7.8 & 0.71 & 0.23 \\
\hline SF-12 (MCS) & $3.7(11.2) \uparrow$ & $0.5(3.4) \uparrow$ & -11.6 to 5.2 & 0.42 & 0.39 \\
\hline Short FES-I & $2.2(3.9) \downarrow$ & $1.8(3.8) \uparrow$ & -0.8 to 8.8 & 0.09 & 1.04 \\
\hline VAS & $1.1(3.0) \downarrow$ & $1.2(2.8) \downarrow$ & -3.6 to 3.4 & 0.95 & 0.03 \\
\hline UPDRS - fall & $0.6(0.8) \downarrow$ & $0.2(0.5) \downarrow$ & -0.6 to 0.3 & 0.39 & 0.59 \\
\hline UPDRS - rigidity & $3.1(3.1) \downarrow$ & $0.8(2.8) \uparrow$ & -3.9 to 0.0 & $0.05^{*}$ & 1.32 \\
\hline UPDRS I & $2.6(2.1) \downarrow$ & $1.8(2.7) \uparrow$ & -7.6 to -1.1 & $<0.01^{*}$ & 1.82 \\
\hline UPDRS II & $7.2(5.5) \downarrow$ & $0.4(3.4) \downarrow$ & -5.9 to -0.5 & $0.02^{*}$ & 1.49 \\
\hline UPDRS III & $16.0(6.2) \downarrow$ & $3.0(5.2) \uparrow$ & -13.2 to -5.7 & $<0.001^{*}$ & 3.30 \\
\hline
\end{tabular}

$\downarrow$ indicates a reduction and $\uparrow$ an increase in each parameter following the treatment. PCS = Physical component summary; MCS = mental component summary. ${ }^{*}$ Significant difference between changes in parameters among two groups after treatment.

Table 5. Mean (SD) and pre-to-post-EA comparison of subjective evaluations and UPDRS parameters for the intervention and the control group

\begin{tabular}{|c|c|c|c|c|c|c|c|c|c|c|}
\hline \multirow[t]{2}{*}{ Parameter } & \multicolumn{5}{|c|}{ Intervention } & \multicolumn{5}{|l|}{ Control } \\
\hline & pre-EA & post-EA & difference & $95 \% \mathrm{CI}$ & $\mathrm{p}$ value & pre-EA & post-EA & difference & $95 \% \mathrm{CI}$ & $\mathrm{p}$ value \\
\hline SF-12 (PCS) & 39.7 (11.7) & $40.3(14.1)$ & $1 \% \uparrow$ & -11.6 to 12.8 & 0.82 & $34.7(9.4)$ & $33.7(5.3)$ & $3 \% \downarrow$ & -12.6 to 10.7 & 0.85 \\
\hline SF-12 (MCS) & $43.4(12.6)$ & $47.1(8.5)$ & $8 \% \uparrow$ & -6.5 to 13.9 & 0.45 & $47.2(7.3)$ & $47.7(7.9)$ & $1 \% \uparrow$ & -10.7 to 11.6 & 0.93 \\
\hline Short FES-I & $17.2(6.8)$ & $14.6(6.9)$ & $15 \% \downarrow$ & -9.0 to 3.8 & 0.41 & $16.6(5.0)$ & $18.4(4.0)$ & $11 \% \uparrow$ & -4.9 to 8.5 & 0.55 \\
\hline VAS & $2.5(3.2)$ & $1.4(2.4)$ & $44 \% \downarrow$ & -3.7 to 1.5 & 0.26 & $3.4(3.3)$ & $2.2(3.0)$ & $35 \% \downarrow$ & -5.8 to 3.4 & 0.50 \\
\hline UPDRS - fall & $0.9(1.3)$ & $0.3(0.7)$ & $67 \% \downarrow$ & -1.6 to 0.4 & $0.02^{*}$ & $0.8(0.8)$ & $0.6(0.5)$ & $25 \% \downarrow$ & -1.3 to 0.9 & 0.37 \\
\hline UPDRS - rigidity & $6.4(5.1)$ & $3.3(2.8)$ & $48 \% \downarrow$ & -8.0 to 0.5 & $0.01^{*}$ & $7.4(4.5)$ & $8.2(4.0)$ & $11 \% \uparrow$ & -6.1 to 8.3 & 0.55 \\
\hline UPDRS I & $5.3(3.5)$ & $2.7(2.3)$ & $49 \% \downarrow$ & -5.5 to 0.6 & $<0.01^{*}$ & $3.3(2.4)$ & $5.1(4.2)$ & $55 \% \uparrow$ & -3.4 to 7.0 & 0.21 \\
\hline UPDRS II & $18.0(9.7)$ & $10.8(6.6)$ & $46 \% \downarrow$ & -10.6 to -3.8 & $<0.0001^{*}$ & $17.0(5.7)$ & $16.6(7.9)$ & $9 \% \uparrow$ & -3.3 to 2.5 & 0.80 \\
\hline UPDRS III & $35.1(15.3)$ & $19.1(10.9)$ & $40 \% \downarrow$ & -12.1 to -19.8 & $<0.01^{*}$ & $34.2(12.3)$ & $37.2(11.6)$ & $2 \% \uparrow$ & -1.6 to 7.6 & 0.27 \\
\hline
\end{tabular}

PCS = Physical component summary; MCS = mental component summary. ${ }^{*}$ Significant difference.

10
Toosizadeh/Lei/Schwenk/Sherman/ Sternberg/Mohler/Najafi 


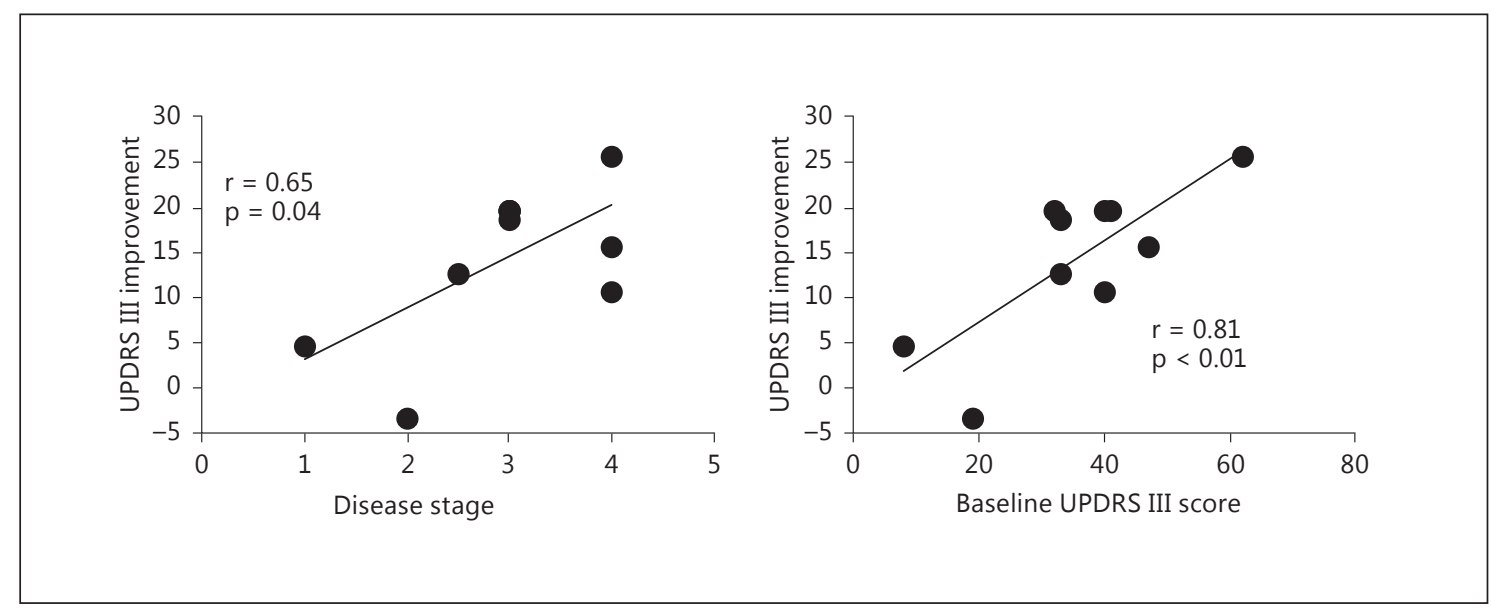

Fig. 4. Correlation between UPDRS III improvement and disease stage, and between UPDRS III improvement and UPDRS III baseline values.

herent structural mechanism of the ankle and hip joints $[41,42]$. The human balancing mechanism activates plantar flexor and dorsiflexor muscles of the ankle and flexor and extensor hip muscles to control body sway in the AP direction, and as such, muscle groups in both legs provide torques in a similar direction. This mechanism helps to overcome the larger flexibility in the AP (compared to the $\mathrm{ML}$ ) direction that occurs because of the hinge-like structure of the ankle and tibiotarsal joints. In contrast, a different controlling mechanism exists to balance the human body in the ML direction using a cancellation strategy. Invertor/evertor muscles of the ankle and abductor/adductor muscles of the hip work in opposite directions on the left and right sides. Therefore, to provide the balance in the ML direction, the controlling mechanism works with loading and unloading of bilateral muscles instead of increasing the level of muscle activity [42]. In this context, the effect of EA treatment was to increase ankle and hip joint compliance during quiet standing, especially in the AP direction. These results were in agreement with clinical evaluation of rigidity here (UPDRS part III-22), which showed a $48 \%$ reduction following EA treatment.

\section{Alterations in Ankle/Hip Sway following EA}

An increased ratio of ankle/hip sway, as expected in our second hypothesis, was evident in the intervention group. In almost all conditions (except eyes-open hip sway), both ankle and hip sway increased following treatment; the amount of increase was, however, larger for ankle joint sway. Accordingly, an increase in ankle/hip sway suggests an increase in using the ankle strategy instead of the hip strategy for maintaining balance following the treatment. A deterioration in using the ankle strategy in PD patients was suggested in previous studies [25], and within the current approach, by using wearable sensors, we were able to accurately evaluate the ankle/hip sway as an indicator of the ratio of using the ankle strategy over the hip strategy. Comparing the PD and the healthy group, we observed that ankle sway was smaller in the PD participants. Accordingly, increased ankle strategy use following EA in the intervention group suggested an improvement in balance. To understand how an increased use of the ankle strategy (instead of the hip strategy) can improve balance, one should consider the differences between these two strategies and their roles in maintaining balance.

To maintain balance on a flat support, healthy individuals dominantly use the ankle strategy. In the condition of a short surface with lack of firmness, or with a lack of sensory feedback, the hip strategy assists in maintaining balance [43]. Similarly, as a result of neuromuscular complications, muscle weakness, or limitation in the range of motion of the ankle joint, larger compensatory motion from the hip and trunk is required to correct the posture during upright standing [44]. Existing evidence indicates that PD patients have altered postural reflexes in their lower extremities, defined by delayed reflex onsets especially in the ankle joints [26, 27]. Additionally, reduced muscle strength and rigidity in the lower extremities, which are associated with PD [21, 45], can lead to smaller ankle strategy use even in simple upright standing on a flat surface. Inability to make full use of the ankles for balancing and a high dependency on upper-body movements therefore may cause 
an increased risk of falling in PD patients [45]. The results following EA demonstrate an increased ankle/hip sway, which may improve balance with the EA intervention. However, it is not clear whether EA improved only rigidity or both rigidity and reflexive responses of the ankles here. In future studies, reflexive responses could be measured using electromyography to address this limitation.

\section{Limitations, Summary of Findings, and Future \\ Directions}

One limitation of the current study is the small number of participants, and, therefore, the current results, while encouraging, should be considered preliminary results which demonstrate the proof of concept for EA benefits to balance in PD patients. These results need to be confirmed in a larger sample in future research. Although balance improvement was observed in almost all individuals in the intervention group, as a result of the small sample size, differences in balance improvement between the intervention and the control group were observed only for ankle/ hip sway. We detected improvements in balance behaviors mostly in the dual-task condition. Based on the minimum estimated effect size here (0.37), to observe a significant improvement in main balance parameters (i.e. $\mathrm{COG}_{\mathrm{ML} / \mathrm{AP}}$ and ankle/hip sway) for the intervention group following EA treatment in the other balance conditions, a sample size of 48 participants is required (power of $80 \%$ and $\alpha=0.05$ ). Similarly, to detect differences in balance improvement between the intervention and the control group in most of the main outcome measures in the eyes-closed and dualtask conditions, a sample size of 51 participants in each group is required. In addition, the improvements in balance behaviors were observed only immediately after EA treatment. The retention of therapy and the long-term effect of acupuncture on balance are therefore still unclear and will be addressed in our future studies. Moreover, we limited the duration of the treatment to 3 weeks ( 3 sessions), and the potential beneficial effect of a longer duration of treatment and gradual improvements in balance should be assessed later. In addition, future research should address the benefit of EA on gait and the reduction of the risk of falling, as well as prospective falls.

A key challenge linked to using wearable sensors to estimate COG is that, unlike camera motion analysis systems or force platforms that use a fixed landmark reference (i.e. room reference axis), the axis of the sensor is highly dependent on how it is attached to the body and how it rotates during body motion. To address this challenge, we used a quaternion model as described earlier [37] to better estimate rotations and correct the changes in landmark refer- ences of the wearable sensors. Further, we used a low-pass filtering algorithm to minimize signal drifting. Another limitation of this study is its use of a two-link invertedpendulum model to estimate COG, assuming that motions around other body joints during a balance test are negligible compared to motions around the hip and ankle joints. In our previous study [46], we demonstrated that this assumption produces an acceptable agreement $(r>0.8)$ for the estimation of the COG trajectory when it is compared to a full biomechanical model of the human body as estimated by a camera motion analysis system.

Despite these limitations, this randomized controlled study provides the proof of concept for potential benefits of non-pharmaceutical-based EA therapy by enhancing balance in aging adults living with PD. Preliminary results revealed that people at advanced stages of $\mathrm{PD}$, with poorer UPDRS III scores, may benefit even more from EA treatment. Our results indicate that the benefit from EA for balance enhancement was independent of the baseline balance in our study. Moreover, a high sensitivity of detecting balance problems was observed for the dual-task condition, which might arise from the inherent neurological sources of PD progression. This was in agreement with UPDRS I subjective evaluations that showed a significant improvement in mentation, behavior, and mood following EA treatment (see tables 4, 5). Previous research also demonstrated an exacerbation of motor performance, specifically during gait, in $\mathrm{PD}$ patients while performing in a dual-task condition [47]. Accordingly, the balance improvement observed here might, to some extent, be related to improvement in cognitive behavior in the PD sample after the treatment. Overall, performing hard cognitive tasks might increase the risk of falling in PD patients, and future studies should aim at studying reasons behind poor balance in dual-task conditions among PD patients and potential exercise regimens for its improvement. In contrast to the dualtask condition for balance testing, small differences were observed in the eyes-open and eyes-closed conditions. Further, UPDRS measures were more sensitive in detecting improvements in PD patients after EA treatment.

\section{Conclusion}

Despite the fact that current conclusions should be confirmed in a larger sample, the current study demonstrates the proof of concept for improvement in PD patients' balance following EA. These improvements are characterized by a reduction in $\mathrm{COG}_{\mathrm{ML} / \mathrm{AP}}$ sway and an enhancement in using the ankle strategy following treatment, which may be
12

Gerontology 2015;61:3-14 DOI: $10.1159 / 000363442$
Toosizadeh/Lei/Schwenk/Sherman/ Sternberg/Mohler/Najafi 
caused by reduced rigidity and increased reflexive responses of the lower limb muscles. These results are in agreement with subjective evaluations of the participants regarding reduced risks of fall and rigidity, and with improvement in motor examination and activities of daily living according to the UPDRS. In these trials the dual task was found to be the most sensitive condition for evaluating enhancement in postural balance following EA treatment. Finally, with the current wearable sensor technology we were able to quantify postural sway in the ankle and hip joints sepa- rately. Using the wearable sensor technology, all measurements were performed in a clinical setting within a short period of time to allow for several measurements.

\section{Acknowledgements}

This study was partially supported by an STTR-Phase II Grant (Award No. 2R42AG032748) from the National Institute on Aging and by the Arizona Center on Aging. We thank Stephan Karp for helping with data collection.

\section{References}

1 Vincent GK, Velkoff VA: The Next Four Decades: The Older Population in the United States: 2010 to 2050. Washington, US Department of Commerce, Economics and Statistics Administration, US Census Bureau, 2010.

- 2 Kannus P, Parkkari J, Koskinen S, Niemi S, Palvanen M, Järvinen M, Vuori I: Fall-induced injuries and deaths among older adults. JAMA 1999;281:1895-1899.

- 3 Stolze H, Klebe S, Zechlin C, Baecker C, Friege L, Deuschl G: Falls in frequent neurological diseases. J Neurol 2004;251:79-84.

4 Thapa PB, Gideon P, Brockman KG, Fought RL, Ray WA: Clinical and biomechanical measures of balance fall predictors in ambulatory nursing home residents. J Gerontol A Biol Sci Med Sci 1996;51:M239-M246.

5 Maki BE, Holliday PJ, Topper AK: A prospective study of postural balance and risk of falling in an ambulatory and independent elderly population. J Gerontol 1994;49:M72-M84.

6 Lajoie Y, Gallagher S: Predicting falls within the elderly community: comparison of postural sway, reaction time, the Berg Balance Scale and the Activities-Specific Balance Confidence $(\mathrm{ABC})$ Scale for comparing fallers and non-fallers. Arch Gerontol Geriatr 2004;38: $11-26$.

7 Dennison AC, Noorigian JV, Robinson KM, Fisman DN, Cianci HJ, Moberg P, BuntingPerry L, Martine R, Duda J, Stern MB: Falling in Parkinson disease: identifying and prioritizing risk factors in recurrent fallers. Am J Phys Med Rehabil 2007;86:621-632.

8 Kerr GK, Worringham CJ, Cole MH, Lacherez PF, Wood JM, Silburn PA: Predictors of future falls in Parkinson disease. Neurology 2010;75:116-124.

9 Horak FB, Frank J, Nutt J: Effects of dopamine on postural control in parkinsonian subjects: scaling, set, and tone. J Neurophysiol 1996;75: 2380-2396

10 Rocchi L, Carlson-Kuhta P, Chiari L, Burchiel KJ, Hogarth P, Horak FB: Effects of deep brain stimulation in the subthalamic nucleus or globus pallidus internus on step initiation in Parkinson disease: laboratory investigation. J Neurosurg 2012;117:1141-1149.
11 St George RJ, Carlson-Kuhta P, Burchiel KJ, Hogarth P, Frank N, Horak FB: The effects of subthalamic and pallidal deep brain stimulation on postural responses in patients with Parkinson disease. J Neurosurg 2012;116: 1347-1356.

12 Allen NE, Sherrington C, Paul SS, Canning CG: Balance and falls in Parkinson's disease: a meta-analysis of the effect of exercise and motor training. Mov Disord 2011;26:16051615.

13 Crizzle AM, Newhouse IJ: Is physical exercise beneficial for persons with Parkinson's disease? Clin J Sport Med 2006;16:422-425.

- 14 Hirsch MA, Toole T, Maitland CG, Rider RA: The effects of balance training and high-intensity resistance training on persons with idiopathic Parkinson's disease. Arch Phys Med Rehabil 2003;84:1109-1117.

15 Gobbi LT, Oliveira-Ferreira MD, Caetano MJD, Lirani-Silva E, Barbieri FA, Stella F, Gobbi S: Exercise programs improve mobility and balance in people with Parkinson's disease. Parkinsonism Relat Disord 2009;15:S49-S52.

16 Lökk J, Nilsson M: Frequency, type and factors associated with the use of complementary and alternative medicine in patients with Parkinson's disease at a neurological outpatient clinic. Parkinsonism Relat Disord 2010;16: 540-544.

17 Rajendran PR, Thompson RE, Reich SG: The use of alternative therapies by patients with Parkinson's disease. Neurology 2001;57:790794.

18 Shulman LM, Wen X, Weiner WJ, Bateman D, Minagar A, Duncan R, Konefal J: Acupuncture therapy for the symptoms of Parkinson's disease. Mov Disord 2002;17:799802.

19 Lee MS, Shin BC, Kong JC, Ernst E: Effectiveness of acupuncture for Parkinson's disease: a systematic review. Mov Disord 2008;23: 1505-1515.

20 Mancini M, Horak FB: The relevance of clinical balance assessment tools to differentiate balance deficits. Eur J Phys Rehabil Med 2010; 46:239.
21 Horak F, Nutt J, Nashner L: Postural inflexibility in parkinsonian subjects. J Neurol Sci 1992;111:46-58.

22 Adkin A, Bloem B, Allum J: Trunk sway measurements during stance and gait tasks in Parkinson's disease. Gait Posture 2005;22:240249 .

23 Ickenstein GW, Ambach H, Klöditz A, Koch $\mathrm{H}$, Isenmann $\mathrm{S}$, Reichmann $\mathrm{H}$, Ziemssen $\mathrm{T}$ : Static posturography in aging and Parkinson's disease. Front Aging Neurosci 2012;4: 20.

24 Paul SS, Canning CG, Sherrington C, Fung VS: Reproducibility of measures of leg muscle power, leg muscle strength, postural sway and mobility in people with Parkinson's disease. Gait Posture 2012;36:639-642.

25 Mitchell S, Collin J, De Luca C, Burrows A, Lipsitz L: Open-loop and closed-loop postural control mechanisms in Parkinson's disease: increased mediolateral activity during quiet standing. Neurosci Lett 1995;197:133-136.

26 Bloem B: Postural instability in Parkinson's disease. Clin Neurol Neurosurg 1992;94:4145.

27 Bloem B, Van Dijk J, Beckley D, Roos R, Remler M, Bruyn G: Altered postural reflexes in Parkinson's disease: a reverse hypothesis. Med Hypoth 1992;39:243-247.

28 Fried LP, Tangen CM, Walston J, Newman AB, Hirsch C, Gottdiener J, Seeman T, Tracy R, Kop WJ, Burke G: Frailty in older adults evidence for a phenotype. J Gerontol A Biol Sci Med Sci 2001;56:M146-M157.

29 Ware JE Jr, Kosinski M, Keller SD: A 12-Item Short-Form Health Survey: construction of scales and preliminary tests of reliability and validity. Med Care 1996;34:220-233.

30 Kempen GI, Yardley L, Van Haastregt JC, Zijlstra GR, Beyer N, Hauer K, Todd C: The Short FES-I: a shortened version of the Falls Efficacy Scale-International to assess fear of falling. Age Ageing 2008;37:45-50.

-31 Langley G, Sheppeard H: The visual analogue scale: its use in pain measurement. Rheumatol Int 1985;5:145-148. 
32 Goetz CG, Tilley BC, Shaftman SR, Stebbins GT, Fahn S, Martinez-Martin P, Poewe W, Sampaio C, Stern MB, Dodel R: Movement disorder society-sponsored revision of the Unified Parkinson's Disease Rating Scale (MDS-UPDRS): scale presentation and clinimetric testing results. Mov Disord 2008;23: 2129-2170.

33 Folstein MF, Folstein SE, McHugh PR: 'Minimental state': a practical method for grading the cognitive state of patients for the clinician. J Psychiatr Res 1975;12:189-198.

-34 Goetz CG, Poewe W, Rascol O, Sampaio C, Stebbins GT, Counsell C, Giladi N, Holloway RG, Moore CG, Wenning GK: Movement disorder society task force report on the Hoehn \& Yahr staging scale: status and recommendations the movement disorder society task force on rating scales for Parkinson's disease. Mov Disord 2004;19:1020-1028.

35 Lam YC, Kum WF, Durairajan SSK, Lu JH, Man SC, Xu M, Zhang XF, Huang XZ, Li M: Efficacy and safety of acupuncture for idiopathic Parkinson's disease: a systematic review. J Altern Complement Med 2008; 14: 663-671.
36 Yeo S, Lim S, Choe IH, Choi YG, Chung KC, Jahng GH, Kim SH: Acupuncture stimulation on GB34 activates neural responses associated with Parkinson's disease. CNS Neurosci Ther 2012;18:781-790.

37 Najafi B, Horn D, Marclay S, Crews RT, Wu S, Wrobel JS: Foot technology, part 1 of 2: assessing postural control and postural control strategy in diabetes patients using innovative and wearable technology. J Diabetes Sci Technol 2010;4:780.

- 38 Najafi B, Bharara M, Talal TK, Armstrong DG: Advances in balance assessment and balance training for diabetes. Diabetes Manag 2012;2:293-308.

39 Gelb DJ, Oliver E, Gilman S: Diagnostic criteria for Parkinson disease. Arch Neurol 1999; 56:33.

40 Stylianou AP, McVey MA, Lyons KE, Pahwa $\mathrm{R}$, Luchies CW: Postural sway in patients with mild to moderate Parkinson's disease. Int J Neurosci 2011;121:614-621.

41 Collins JJ, De Luca CJ: Open-loop and closedloop control of posture: a random-walk analysis of center-of-pressure trajectories. Exp Brain Res 1993;95:308-318.
42 Winter DA: Human balance and posture control during standing and walking. Gait Posture 1995;3:193-214.

43 Runge C, Shupert C, Horak F, Zajac F: Ankle and hip postural strategies defined by joint torques. Gait Posture 1999;10:161-170.

44 Horak FB: Clinical measurement of postural control in adults. Phys Ther 1987;67:18811885.

45 Bloem BR, Van Vugt J, Beckley DJ: Postural instability and falls in Parkinson's disease. Adv Neurol 2001;87:209.

46 Najafi B, Lee-Eng J, Marclay S, Wrobel J, Goebel R: Estimation of center of mass trajectory using wearable sensors during golf swing. Sensors 2014, under review.

47 O'Shea S, Morris ME, Iansek R: Dual task interference during gait in people with Parkinson disease: effects of motor versus cognitive secondary tasks. Phys Ther 2002;82:888-897. 